\title{
ON SUBORDINATION FOR CERTAIN SUBCLASS OF ANALYTIC FUNCTIONS
}

\author{
LIU JINLIN \\ Water Conservancy College \\ Yangzhou University \\ Yangzhou 225009, P.R CHINA
}

(Received August 17, 1995 and in revised form July 9, 1996)

ABSTRACT. In the present paper the class $P_{n}[\alpha, M]$ consisting of functions $f(z)=z+\sum_{k=n+1}^{\infty} a_{k} z^{k}(n \geq 1)$, which are analytic in the unit disc $E=\{z:|z|<1\}$ and satisfy the condition $\left|f^{\prime}(z)+\alpha z f^{\prime \prime}(z)-1\right|<M$ is introduced. By using the method of differential subordination the properties of the class $P_{n}[\alpha, M]$ are discussed.

KEY WORDS AND PHRASES: Analytic, starlike, convex univalent, subordination 1991 AMS SUBJECT CLASSIFICATION CODES: 30C45

\section{INTRODUCTION}

Let $A_{n}(n \geq 1)$ denote the class of functions of the form $f(z)=z+\sum_{k=n+1}^{\infty} a_{k} z^{k}$ which are analytic in the unit disc $E=\{z:|z|<1\}$. A function $f(z)$ in $A_{n}$ is said to be in $P_{n}[\alpha, M]$ for some $\alpha(\alpha \geq 0)$ and $M(M>0)$ if it satisfies the condition

$$
\left|f^{\prime}(z)+\alpha z f^{\prime \prime}(z)-1\right|<M(z \in E) .
$$

Let $f(z)$ and $g(z)$ be analytic in $E$. Then we say that the function $g(z)$ is subordinate to $f(z)$ in $E$ if there exists an analytic function $w(z)$ in $E$ such that $|w(z)|<1(z \in E)$ and $g(z)=f(w(z))$ For this relation the symbol $g(z) \prec f(z)$ is used. In case $f(z)$ is univalent in $E$ we have that the subordination $g(z) \prec f(z)$ is equivalent to $g(0)=f(0)$ and $g(E) \subset f(E)$.

In this paper, we shall use the method of differential subordination [2] to obtain certain properties of the class $P_{n}[\alpha, M]$.

\section{MAIN RESULTS}

In order to give our main results, we need the following lemma.

LEMMA [1]. Let $p(z)=a+p_{n} z^{n}+\ldots(n \geq 1)$ be analytic in $E$ and let $h(z)$ be convex univalent in $E$ with $h(0)=a$. If $p(z)+\frac{1}{c} z p^{\prime}(z) \prec h(z)$, where $c \neq 0$ and $\operatorname{Re} c \geq 0$, then $p(z) \prec \frac{c}{n} z^{-\frac{\varepsilon}{n}} \int_{0}^{z} h(t) t^{\frac{\varepsilon}{n}-1} d t$

Applying the above lemma, we derive

THEOREM 1. Let $f(z) \in P_{n}[\alpha, M]$, then

$$
\begin{gathered}
\left|f^{\prime}(z)\right| \leq 1+\frac{M}{1+n \alpha}|z|^{n}, \\
\operatorname{Re} f^{\prime}(z) \geq 1-\frac{M}{1+n \alpha}|z|^{n},
\end{gathered}
$$




$$
\begin{gathered}
|f(z)| \leq|z|+\frac{M}{(1+n)(1+n \alpha)}|z|^{n+1}, \\
\operatorname{Re} f(z) \geq|z|-\frac{M}{(1+n)(1+n \alpha)}|z|^{n+1} .
\end{gathered}
$$

The results are sharp.

PROOF. Since $f(z) \in P_{n}[\alpha, M]$, it follows from (1.1) that

$$
f^{\prime}(z)+\alpha z f^{\prime \prime}(z) \prec 1+M z .
$$

With the help of the lemma, (2.5) yields

$$
f^{\prime}(z) \prec \frac{1}{n \alpha} z^{-\frac{1}{n \alpha}} \int_{0}^{z}(1+M t) t^{\frac{1}{n a}-1} d t=1+\frac{M}{1+n \alpha} z .
$$

Using (2.6), we get

$$
f^{\prime}(z)=1+\frac{M}{1+n \alpha} w(z),
$$

where $w(z)$ is analytic in $E$ and $|w(z)| \leq|z|^{n}$. Thus, from (2.7) we obtain (2.1) and (2.2) immediately.

Further, using (2.1) and (2.2) we can arrive at (2.3) and (24) by integration, as follows

$$
\begin{aligned}
f(z)= & \int_{0}^{z} f^{\prime}(t) d t=\int_{0}^{|z|} f^{\prime}\left(t e^{\imath \Theta}\right) e^{\imath \Theta} d t \\
|f(z)| & \leq \int_{0}^{|z|}\left|f^{\prime}\left(t e^{i \Theta}\right)\right| d t \\
& \leq \int_{0}^{|z|}\left(1+\frac{M}{1+n \alpha} t^{n}\right) d t=|z|+\frac{M}{(1+n)(1+n \alpha)}|z|^{n+1}, \\
\operatorname{Re} f(z) & \geq \int_{0}^{|z|} \operatorname{Re} f^{\prime}\left(t e^{2 \Theta}\right) d t \\
& \geq \int_{0}^{|z|}\left(1-\frac{M}{1+n \alpha} t^{n}\right) d t=|z|-\frac{M}{(1+n)(1+n \alpha)}|z|^{n+1} .
\end{aligned}
$$

By considering the function

$$
f(z)=z+\frac{M}{(1+n)(1+n \alpha)} z^{n+1},
$$

we can show that all estimates of this theorem are sharp.

According to the proof of Theorem 1, we have

COROLLARY. Let $f(z) \in P_{n}[\alpha, M]$, then

$$
\begin{gathered}
\left|f^{\prime}(z)-1\right|<\frac{M}{1+n \alpha}, \\
\left|\frac{f(z)}{z}-1\right|<\frac{M}{(1+n)(1+n \alpha)} .
\end{gathered}
$$

The results are sharp.

THEOREM 2. Let $f(z) \in P_{n}[\alpha, M]$. If $M \leq 1+n \alpha$, then $\operatorname{Re}\left\{e^{\imath \beta} f^{\prime}(z)\right\}>0(z \in E)$, where $\beta$ is real and $|\beta| \leq \frac{\pi}{2}-\operatorname{arc} \sin \frac{M}{1+n \alpha}|z|^{n}$. The result is sharp in the sense that the range of $\beta$ cannot be increased.

PROOF. From the proof of Theorem 1, we have 


$$
\left|\arg \left\{e^{2 \beta} f^{\prime}(z)\right\}\right| \leq|\beta|+\left|\arg f^{\prime}(z)\right| \leq|\beta|+\arcsin \frac{M}{1+n \alpha}|z|^{n} \leq \frac{\pi}{2}
$$

for $|\beta| \leq \frac{\pi}{2}-\arcsin \frac{M}{1+n \alpha}|z|^{n}$

The result is sharp and the extremal function has the form of (2.8)

THEOREM 3. Let $f(z) \in P_{n}[\alpha, M]$ If $M \leq \frac{(1+n)(1+n \alpha)}{\sqrt{1+(1+n)^{2}}}$, then $f(z)$ is univalent starlike in $E$

PROOF. According to the corollary and the assumption of Theorem 3 , it follows immediately that $\operatorname{Re} f^{\prime}(z)>0(z \in E)$ and $\operatorname{Re} \frac{f(z)}{z}>0(z \in E)$

On the other hand, we see that

$$
\left|\arg f^{\prime}(z)\right|<\arcsin \frac{M}{1+n \alpha} \leq \arcsin \frac{1+n}{\sqrt{1+(1+n)^{2}}},
$$

and

$$
\left|\arg \frac{f(z)}{z}\right|<\arcsin \frac{M}{(1+n)(1+n \alpha)} \leq \arcsin \frac{1}{\sqrt{1+(1+n)^{2}}} .
$$

Using (2.11) and (2.12), we obtain

$$
\begin{aligned}
\left|\arg \frac{z f^{\prime}(z)}{f(z)}\right| & \leq\left|\arg f^{\prime}(z)\right|+\left|\arg \frac{f(z)}{z}\right| \\
& <\arcsin \frac{1+n}{\sqrt{1+(1+n)^{2}}}+\arcsin \frac{1}{\sqrt{1+(1+n)^{2}}} \\
& =\frac{\pi}{2} \quad(z \in E)
\end{aligned}
$$

which implies that $f(z)$ is univalent starlike in $E$.

THEOREM 4. Let $c>-1$ and let $f(z) \in P_{n}[\alpha, M]$. Then the function $F(z)$ defined by

$$
F(z)=\frac{c+1}{z^{c}} \int_{0}^{z} t^{c-1} f(t) d t
$$

belongs to $P_{n}\left[\frac{1}{c+1}, \frac{M}{1+n \alpha}\right]$. The result is sharp.

PROOF. By (2.13) and (2.6), we have

$$
F^{\prime}(z)+\frac{1}{c+1} z F^{\prime \prime}(z)=f^{\prime}(z) \prec 1+\frac{M}{1+n \alpha} z,
$$

which shows that $F(z) \in P_{n}\left[\frac{1}{c+1}, \frac{M}{1+n \alpha}\right]$

This result is sharp and the extremal function has the form of (2.8).

THEOREM 5. Let $c>-1$ and $\alpha>0$. If $F(z) \in P_{n}[\alpha, M]$, then the function $f(z)$ defined by (2.13) satisfies $\left|f^{\prime}(z)-1\right|<M$ for $z \in E$.

PROOF. Since $F(z) \in P_{n}[\alpha, M]$, we have from (1.1), (2.5) and (2.6) that

$$
F^{\prime}(z)+\alpha z F^{\prime \prime}(z) \prec 1+M z
$$

and

$$
F^{\prime}(z) \prec 1+\frac{M}{1+n \alpha} z .
$$

From (2.13), we get

$$
f^{\prime}(z)=\frac{1}{\alpha(c+1)}\left\{\left[F^{\prime}(z)+\alpha z F^{\prime \prime}(z)\right]+[\alpha(c+1)-1] F^{\prime}(z)\right\}
$$


On using (2 14) and (2.15), (2.16) yields

$$
\begin{aligned}
f^{\prime}(z) & =\frac{1}{\alpha(c+1)}\left\{\left[F^{\prime}(z)+\alpha z F^{\prime \prime}(z)\right]+[\alpha(c+1)-1] F^{\prime}(z)\right\} \\
& \prec \frac{1}{\alpha(c+1)}\{1+M z+[\alpha(c+1)-1](1+M z)\} \\
& =1+M z
\end{aligned}
$$

which implies that $\left|f^{\prime}(z)-1\right| \leq M|z|<M(z \in E)$.

ACKNOWLEDGMENT. The author expresses his grateful thanks to the referee for his useful suggestions

\section{REFERENCES}

[1] MILLER, S S. and MOCANU, P.T., Differential subordinations and univalent functions, Michigan Math. J. 28 (1981), 157-171.

[2] MILLER, S.S. and MOCANU, P.T., On some classes of first-order differential subordinations, Michigan Math. J. 32 (1985), 185-195. 


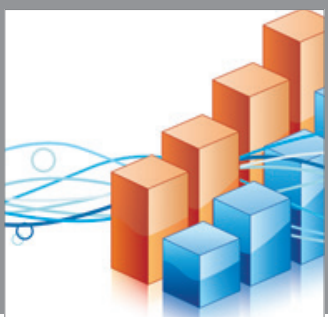

Advances in

Operations Research

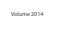

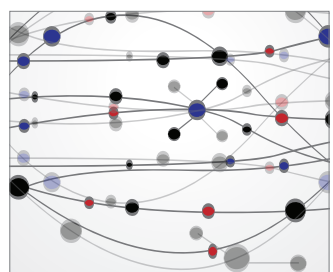

\section{The Scientific} World Journal
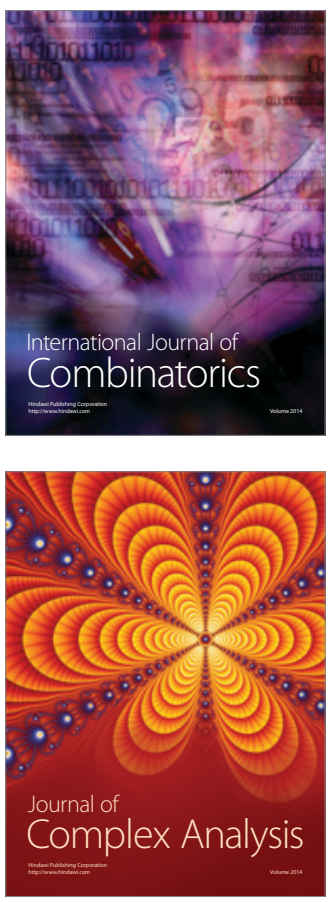

International Journal of

Mathematics and

Mathematical

Sciences
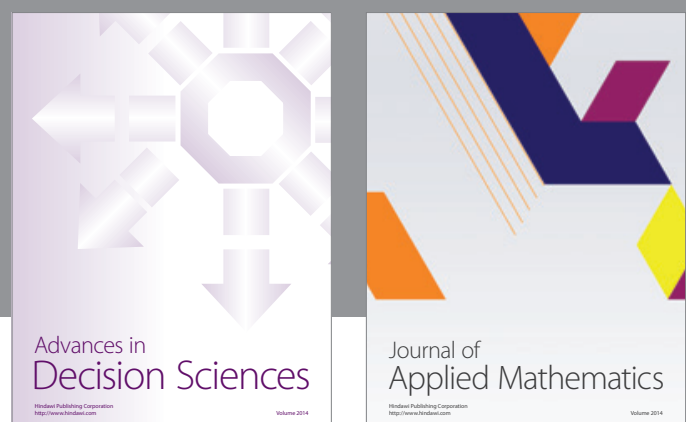

Journal of

Applied Mathematics
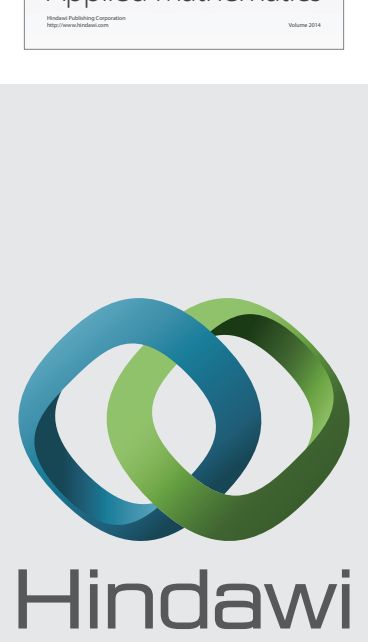

Submit your manuscripts at http://www.hindawi.com
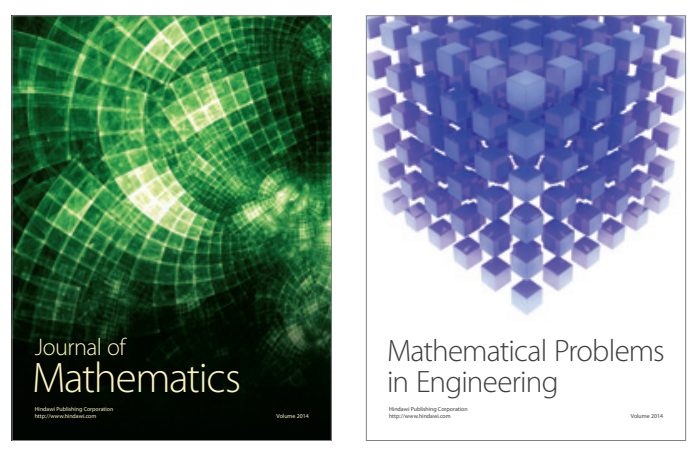

Mathematical Problems in Engineering
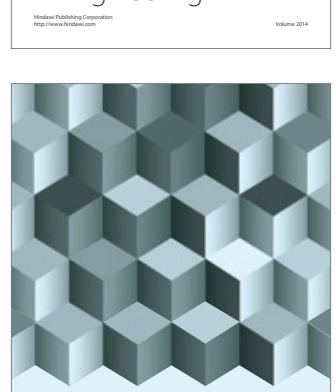

Journal of

Function Spaces
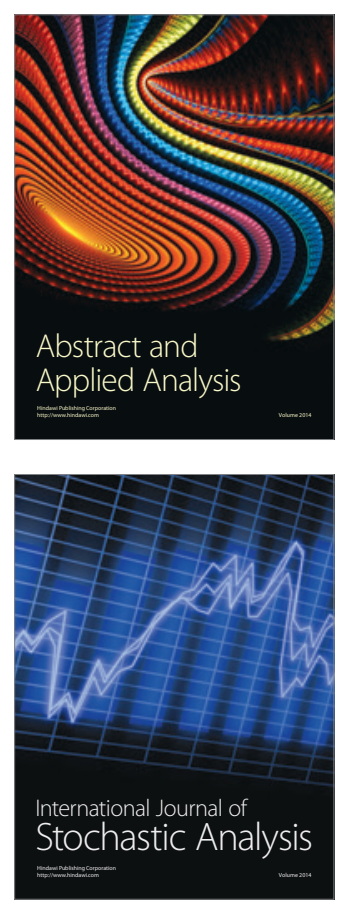

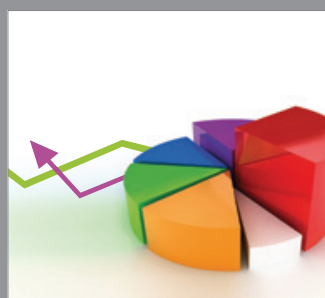

ournal of

Probability and Statistics

Promensencen
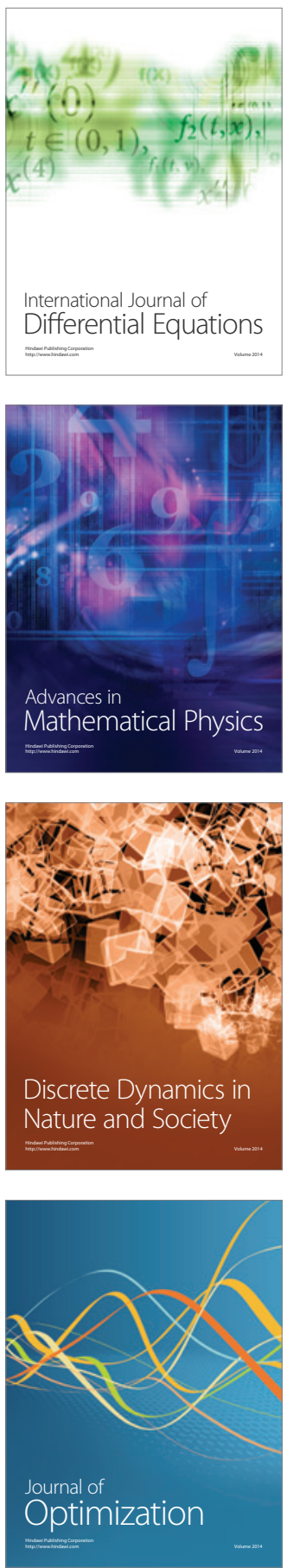